\title{
On Green's Functions, Propagators, and Sturmians for the Nonrelativistic Coulomb Problem
}

\author{
S. M. BLINDER \\ Department of Chemistry, University of Michigan, Ann Arbor, Michigan 48109
}

\begin{abstract}
Recent progress in the mathematical physics and quantum chemistry of Coulomb Green's functions is summarized. Analogy with the defining relation for the Green's function has led to a finite model for the Fermi contact interaction which avoids spurious divergences in second-order perturbation calculations. The Hamilton-Jacobi mechanics of the Coulomb problem is reviewed. A compact parametrization for Hamilton's principal and characteristic functions provides a key element in further developments. These include a semiclassical representation for the Coulomb propagator in Feynman's formalism and a new propagator in the domain of Coulomb Sturmian eigenstates. In projected applications, approximate many-electron Green's functions constructed from combinations of oneparticle Coulomb propagators provide a basis for computation of atomic and molecular eigenvalue spectra.
\end{abstract}

\section{Coulomb Green's Functions}

The hydrogen atom has played a key historical role in the development of the quantum theory of matter, from the Bohr model to Schrödinger's wave mechanics to Dirac's relativistic theory to modern quantum electrodynamics to current models exploiting higher dynamical symmetry. The literature on Coulomb Green's functions has been correspondingly extensive, dating back to Meixner's partial solution for $G\left(\mathbf{r}_{1}, \mathbf{r}_{2}, E\right)$ in the limits $r_{2}=0$ or $\infty$ [1]. A number of integral representations and partial-wave expansions for the time-independent Coulomb Green's function were subsequently developed [2]. Schwinger [3] gave an elegant representation for the Green's function in momentum space. Hostler [4] finally discovered a closed form for the Coulomb Green's function $G\left(\mathbf{r}_{1}, \mathbf{r}_{2}, E\right)$ and also derived approximate relativistic Green's functions for both the Klein-Gordon and Dirac equations.

The Coulomb Green's function is the solution under specified boundary conditions of the inhomogeneous differential equation

$$
\left(E+\frac{1}{2} \nabla_{1}^{2}+Z / r_{1}\right) G\left(\mathbf{r}_{1}, \mathbf{r}_{2}, E\right)=\delta\left(\mathbf{r}_{1}-\mathbf{r}_{2}\right)
$$

in terms of atomic units, $\hbar=e=m=1$. Hostler's solution for the retarded (outgoing wave) Green's function can be written [5]

$$
G^{+}\left(\mathbf{r}_{1}, \mathbf{r}_{2}, E\right)=G^{+}(x, y, k)=-\frac{1}{\pi(x-y)}\left(\frac{\partial}{\partial x}-\frac{\partial}{\partial y}\right) g^{+}(x, y, k)
$$


where

$$
g^{+}(x, y, k)=(i k)^{-1} \Gamma(1-i v) M_{i v}^{1 / 2}(-i k y) W_{i v}^{1 / 2}(-i k x)
$$

in terms of the variables

$$
x \equiv r_{1}+r_{2}+r_{12}, \quad y \equiv r_{1}+r_{2}-r_{12}
$$

and the parameters $k$ and $v$, defined such that

$$
E=\hbar^{2} k^{2} / 2 m, \quad v=Z / k a_{0}, \quad \operatorname{Im} k>0 ;
$$

$M$ and $W$ represent Whittaker functions of the first and second kind, as defined by Buchholz [6]:

$$
\begin{aligned}
M_{i \nu}^{m / 2}(-i k y)= & (m !)^{-1}(-i k y)^{(m+1) / 2} e^{-i k y / 2} F_{1}\left(\frac{1}{2}(m+1)+i \nu ; m+1 ; i k y\right) \\
M_{i \nu}^{m / 2}(-i k x)= & e^{-\pi \nu} \\
& \times\left(\frac{W_{-i \nu}^{m / 2}(i k x)}{\Gamma\left(\frac{1}{2}(m+1)-i \nu\right)}+e^{-i(m+1) \pi / 2} \frac{W_{i \nu}^{m / 2}(-i k x)}{\Gamma\left(\frac{1}{2}(m+1)+i v\right)}\right)
\end{aligned}
$$

where ${ }_{1} F_{1}$ is the confluent hypergeometric function

$$
{ }_{1} F_{1}(a ; b ; z) \equiv M(a, b, z)=\sum_{n=0}^{\infty} \frac{(a)_{n}}{(b)_{n}} \frac{z^{n}}{n !} .
$$

Remarkably, the Coulomb Green's function depends on just two independent coordinates, the combinations $x$ and $y$, rather than the three variables, say $r_{1}$, $r_{2}, r_{12}$, which would be implied by the spherical symmetry of the Hamiltonian. This reduction is now understood to be a consequence of the higher dynamical symmetry of the Coulomb problem, specifically represented by the groups $\mathrm{SO}(4)$ or $\mathrm{SO}(3,1)$ for bound and continuum states, respectively [7]. As a concomitant, there exists an additional constant of the motion-the Runge-Lenz vector [8]. The significance of the variables $x$ and $y$ is discussed below.

In its dependence on two particle coordinates, the Coulomb Green's function somewhat resembles a helium wave function. In a variational calculation on helium using a function of the form

$$
\psi(x, y)=\sum_{m, n} C_{m, n} x^{m} y^{n} e^{-\alpha(x+y)}
$$

an energy of -2.89175065 hartrees was obtained, accounting for $71.5 \%$ of the correlation energy [9]. This is, in fact, the most accurate calculation to date based on a two-variable helium wave function. The wave function (9) would be equivalent to a Hylleraas-type function [10] with the variable $t$ of the trio $s, t$, $u$ absent.

\section{A Model for the Fermi Contact Interaction}

Interactions between s electrons and magnetic nuclei are well accounted for by the Fermi contact operator [11]

$$
H^{\prime}=\frac{8}{3} \pi g g_{\mathrm{I}} \mu_{B} \mu_{N} \mathbf{S} \cdot \mathbf{I} \delta(\mathbf{r}), \quad \delta(\mathbf{r})=\delta(r) / 4 \pi r^{2}
$$


Atomic hyperfine structure and nuclear spin-spin coupling have been successfully treated using this delta-function operator. Infinite terms arise, however, when this operator is applied beyond first order in perturbation theory [12]. To eliminate these spurious divergences, we have suggested a modified version of the Fermi contact interaction in which the point nuclear magnetic dipole implied by (10) is replaced by a uniformly magnetized spherical shell of radius $r_{0}$. This is effected by the substitution in Eq. (10) of $\delta\left(r-r_{0}\right)$ for $\delta(r)$, in which $r_{0}$ is of the order of a nuclear diameter [13]. A perturbed hydrogenic atom in an $s$ state is thus represented by the Schrödinger equation

$$
\begin{array}{r}
{\left[E+\frac{1}{2} \nabla^{2}+Z / r-\left(\lambda / 4 \pi r_{0}^{2}\right) \delta\left(r-r_{0}\right)\right] \psi(r)=0,} \\
\lambda \equiv \frac{8}{3} \pi g g_{1} \mu_{B} \mu_{N} \mathbf{S} \cdot \mathbf{I} .
\end{array}
$$

With introduction of the radial function such that $\psi(r)=\left(4 \pi r^{2}\right)^{-1 / 2} P(r)$, Eq. (11) reduces to

$$
\left(E+\frac{1}{2} \frac{\partial^{2}}{\partial r^{2}}+\frac{Z}{r}\right) P(r)=\frac{\lambda}{4 \pi r_{0}^{2}} P\left(r_{0}\right) \delta\left(r-r_{0}\right) .
$$

Equation (12) is isomorphous with the $S$-wave component of (1), the defining equation for the Coulomb Green's function. Specifically,

$$
\left(\frac{1}{2} k^{2}+\frac{\partial^{2}}{\partial r^{2}}+\frac{Z}{r}\right) g\left(r, r_{0}, k\right)=\delta\left(r-r_{0}\right)
$$

with $g\left(r, r_{0}, k\right)$ equal to (3) after the substitutions $x \rightarrow 2 r, y \rightarrow 2 r_{0}$. Since we are interested in bound states, it is expedient to make the further substitutions $k \rightarrow i k$, $v \rightarrow-i v$. One obtains thereby the eigenfunctions of the Schrödinger equation (12):

$$
P_{\nu}(r)=-\left(\lambda / 4 \pi k r_{0}^{2}\right) P_{\nu}\left(r_{0}\right) \Gamma(1-\nu) M_{\nu}^{1 / 2}\left(2 k r_{<}\right) W_{\nu}^{1 / 2}\left(2 k r_{>}\right) .
$$

The corresponding eigenvalues, given by

$$
E=-\frac{1}{2} k^{2}=-Z^{2} / 2 v^{2},
$$

are determined by the transcendental equation

$$
-\left(\lambda / 4 \pi k r_{0}^{2}\right) \Gamma(1-v) M_{\nu}^{1 / 2}\left(2 k r_{0}\right) W_{v}^{1 / 2}\left(2 k r_{0}\right)=1 .
$$

The energies (15) have the same form as unperturbed Coulomb eigenvalues but with nonintegral values of the quantum number $v$. Since the perturbation on the atomic state is exceedingly small $\left(\lambda \sim 10^{-7}\right.$ for hydrogen), it is convenient to introduce a quantum defect such that $v=n+\delta$. The energies (15) are correspondingly approximated by

$$
E=-Z^{2} / 2 n^{2}+Z^{2} \delta / n^{3}+O\left(\delta^{2}\right)
$$

where it follows from (14) that $\delta \approx \lambda Z$, provided that $r_{0}$ is of nuclear dimension. A useful result is the first-order approximation for a 1 s orbital with effective nuclear charge $\zeta$ :

$$
\psi_{1 s}(r)=W_{1+\delta}(r) / r \approx e^{-\zeta r}[1+\delta(-1+\zeta r+\ln 2 \zeta r-1 / 2 \zeta r)] .
$$


The limiting behavior as $r_{0}$ approaches 0 has been discussed in detail in the original references. It reveals the explicit nature of the divergences associated with the Fermi contact interaction. As $r_{0} \rightarrow 0$, the conventional Coulomb plus delta-function potential is recovered. The magnetic perturbation energy goes to zero for a repulsive delta function, but approaches $-\infty$ for an attractive delta function. In subsequent work, a quark model for the proton has been applied in a precise calculation of the hyperfine splitting in atomic hydrogen [14].

The modified Fermi potential has been applied to NMR spin-spin coupling in the HD molecule [15]. Perturbed 1s atomic orbitals of the form (18) were used to construct Heitler-London molecular wave functions. Since such functions already account for magnetic interactions with one of the nuclei, the computation requires only first-order perturbation theory. Thus the divergences that have long plagued this problem [16] are entirely avoided. The coupling constant $J_{\mathrm{HD}}$ is identified as the part of the energy proportional to $\mathbf{I}_{\mathrm{H}} \cdot \mathbf{I}_{\mathrm{D}}$. After averaging over zero-point vibration, one obtains a coupling constant of $41.96 \mathrm{~Hz}$ via the contact interaction. Adding $0.30 \mathrm{~Hz}$ from the spin-dipolar and orbital contributions [17], we obtain $J_{\mathrm{HD}}=42.26 \mathrm{~Hz}$, in remarkable agreement with the experimental value, $42.94 \pm 0.10 \mathrm{~Hz}$.

\section{Generalized Unsöld Theorem}

The Coulomb Green's function has the following spectral representation:

$$
G\left(\mathbf{r}_{1}, \mathbf{r}_{2}, E\right)=\sum_{n l m} \frac{\psi_{n l m}\left(\mathbf{r}_{1}\right) \psi_{n l m}^{*}\left(\mathbf{r}_{2}\right)}{E-E_{n}}
$$

with $\operatorname{Im} E>0$ for $G^{+}$. The summation runs over both discrete and continuum eigenstates. For reference purposes, we give the hydrogenic eigenfunctions expressed in terms of Whittaker functions:

$\psi_{n l m}(\mathbf{r})=\left(\frac{k}{n}\right)^{1 / 2}\left(\frac{(n+l) !}{(n-l-1) !}\right)^{1 / 2} r^{-1} M_{n}^{l+1 / 2}(2 k r) Y_{l m}(\theta, \phi), \quad k \equiv Z / n$.

The corresponding continuum eigenfunctions are

$$
\begin{array}{r}
\psi_{\nu l m}(\mathbf{r})=(2 \pi)^{1 / 2} i^{l+1}(k / \nu)^{1 / 2} e^{\pi \nu / 2} \Gamma(l+1-i \nu) r^{-1} M_{i v}^{l+1 / 2}(-2 i k r) Y_{l m}(\theta, \phi), \\
k \equiv Z / \nu, 0 \leqslant v<\infty .
\end{array}
$$

Since hydrogenic energy eigenvalues depend only on the principal quantum number $n$, the residue at each pole, where $E=-Z^{2} / 2 n^{2}$, contains a sum over compatible values of 1 and $m$. We define the density function

$$
\rho_{n}\left(\mathbf{r}_{1}, \mathbf{r}_{2}\right)=\sum_{l=0}^{n-1} \sum_{m=-l}^{l} \psi_{n l m}\left(\mathbf{r}_{1}\right) \psi_{n ! m}^{*}\left(\mathbf{r}_{2}\right)
$$

We now make use of the Green's function (2)-(3) and note the reduction of both Whittaker functions to Laguerre functions for integer values of the lower index (cf. [6, p. 214]). Retaining the Whittaker functions $M$ for compact notation, we 
obtain [18]

$$
\rho_{n}\left(\mathbf{r}_{1}, \mathbf{r}_{2}\right)=-\left(\frac{Z}{n \pi}\right)(x-y)^{-1}\left(\frac{\partial}{\partial x}-\frac{\partial}{\partial y}\right) M_{n}^{1 / 2}\left(\frac{Z x}{n}\right) M_{n}^{1 / 2}\left(\frac{Z y}{n}\right) .
$$

This result represents a generalization of Unsöld's theorem [19], which pertains to a sum over $m$ with fixed values of both 1 and $n$. Explicitly, the first three density functions are given by

$$
\begin{aligned}
\rho_{1} & =\left(Z^{3} / \pi\right) e^{-Z \xi}, \\
\rho_{2} & =\left(Z^{3} / 8 \pi\right)\left(1-\frac{1}{2} Z \xi+\frac{1}{8} Z^{2} \eta^{2}\right) e^{-Z \xi / 2}, \\
\rho_{3} & =\left(Z^{3} / 27 \pi\right)\left[1-\frac{2}{3} Z \xi+\frac{2}{27} Z^{2}\left(\xi^{2}+2 \eta^{2}\right)\right. \\
& \left.-\frac{2}{81} Z^{3} \xi \eta^{2}+\frac{1}{972} Z^{4} \eta^{4}\right] e^{-Z \xi / 3}, \\
\xi & \equiv \frac{1}{2}(x+y), \eta \equiv(x y)^{1 / 2} .
\end{aligned}
$$

Applications to the study of hybrid atomic orbitals and open-shell configurations are projected.

The analog of Eq. (23) for continuum eigenstates works out to

$$
\begin{aligned}
\sum_{l, m} \psi_{v l m}\left(\mathbf{r}_{1}\right) \psi_{\nu l m}^{*}\left(\mathbf{r}_{2}\right)=\frac{k}{2 \pi^{2} v} e^{\pi \nu}|\Gamma(1-i v)|^{2}(x-y)^{-1}\left(\frac{\partial}{\partial x}-\frac{\partial}{\partial y}\right) \\
\times M_{i v}^{1 / 2}(-i k x) M_{i \nu}^{1 / 2}(-i k y), \quad k=\frac{Z}{\nu}, E=\frac{Z^{2}}{2 v^{2}}, 0 \leqslant v<\infty .
\end{aligned}
$$

\section{Hamilton-Jacobi Mechanics for the Coulomb Problem}

A necessary adjunct to some important further developments on Coulomb propagators involves the classical Hamilton-Jacobi theory of the Kepler-Coulomb problem [20]. We begin with the time-independent equation for Hamilton's characteristic function $W\left(\mathbf{r}_{1}, \mathbf{r}_{2}, E\right)$ :

$$
\frac{1}{2}\left(\nabla_{1} W\right)^{2}-Z / r_{1}=E
$$

with an analogous equation in $\mathbf{r}_{2}$. We interpret $\mathbf{r}_{1}$ and $\mathbf{r}_{2}$ as the initial and final displacements, respectively, in a classically allowed orbit. The orbital angular momentum is a constant of the motion; thus

$$
\mathbf{L}=\mathbf{r}_{1} \times \mathbf{p}_{1}=\mathbf{r}_{2} \times \mathbf{p}_{2}=-\mathbf{r}_{1} \times \nabla_{1} W=\mathbf{r}_{2} \times \nabla_{2} W .
$$

We can write

$$
\nabla_{1} W=\hat{\mathbf{u}}_{1} \frac{\partial W}{\partial r_{1}}+\hat{\mathbf{u}}_{12} \frac{\partial W}{\partial r_{12}}, \quad \nabla_{2} W=\hat{\mathbf{u}}_{2} \frac{\partial W}{\partial r_{2}}+\hat{\mathbf{u}}_{12} \frac{\partial W}{\partial r_{12}}
$$

in terms of the nonorthogonal unit vectors

$$
\hat{\mathbf{u}}_{1} \equiv \frac{\mathbf{r}_{1}}{r_{1}}, \quad \hat{\mathbf{u}}_{2} \equiv \frac{\mathbf{r}_{2}}{r_{2}}, \quad \hat{\mathbf{u}}_{12} \equiv \frac{\mathbf{r}_{12}}{r_{12}}, \quad \mathbf{r}_{12} \equiv \mathbf{r}_{1}-\mathbf{r}_{2}
$$

$$
r_{12}=\left|\mathbf{r}_{1}-\mathbf{r}_{2}\right|
$$


It follows that

$$
\mathbf{L}=\frac{\mathbf{r}_{1} \times \mathbf{r}_{2}}{r_{12}} \frac{\partial W}{\partial r_{12}}=\hat{\mathbf{u}}_{1} \times \hat{\mathbf{u}}_{2} \frac{r_{1} r_{2}}{r_{12}} \frac{\partial W}{\partial r_{12}} .
$$

The constancy of $\mathbf{L}$ implies that $W$ can be expressed as a function of the three variables $r_{1}, r_{2}, r_{12}$. A further reduction is made possible by the existence of an additional constant of the motion, the Runge-Lenz vector [21]

$$
\mathbf{A} \equiv\left(Z e^{2} m\right)^{-1} \mathbf{L} \times \mathbf{p}+\mathbf{r} / r
$$

In terms of $W$,

$$
\mathbf{A}=-Z^{-1} \mathbf{L} \times \nabla_{1} W+\hat{\mathbf{u}}_{1}=Z^{-1} \mathbf{L} \times \nabla_{2} W+\hat{\mathbf{u}}_{2} .
$$

The scalar product with $\hat{\mathbf{u}}_{1}+\hat{\mathbf{u}}_{2}$ results in

$$
\mathbf{L} \times\left(\nabla_{1} W+\nabla_{2} W\right) \cdot\left(\hat{\mathbf{u}}_{1}+\hat{\mathbf{u}}_{2}\right)=0 .
$$

Thus, using (29) and (30), we have

$$
\frac{\partial W}{\partial r_{1}}-\frac{\partial W}{\partial r_{2}}=0
$$

This shows that $W$ is independent of the variable $r_{1}-r_{2}$. It is thus a function of just the two variables $r_{1}+r_{2}$ and $r_{12}$. Cross products of derivatives are avoided if one chooses as new independent variables the linear combinations

$$
x \equiv r_{1}+r_{2}+r_{12}, \quad y \equiv r_{1}+r_{2}-r_{12}, \quad x \geqslant y \geqslant 0 .
$$

After some algebra, the Hamilton-Jacobi (HJ) equation (26) reduces to

$$
W_{x}^{2}-Z / x=\frac{1}{2} E, \quad W_{y}^{2}-Z / y=\frac{1}{2} E .
$$

The transformed $\mathrm{HJ}$ equations (36) have the form of a quasi-one-dimensional problem with $y$ and $x$ representing the initial and final coordinates, respectively. The differential equations are simplified by introduction of the auxilliary variables $\lambda, \mu$, and $\nu$, whereby

$$
E=\frac{Z^{2}}{2 v^{2}}, \quad \sinh \lambda=\left(\frac{Z x}{4 v^{2}}\right)^{1 / 2}, \quad \sinh \mu=\left(\frac{Z y}{4 v^{2}}\right)^{1 / 2} .
$$

Equations (36) thereby reduce to

$$
W_{\lambda}= \pm 4 \nu \cosh ^{2} \lambda, \quad W_{\mu}= \pm 4 \nu \cosh ^{2} \mu .
$$

A solution for Hamilton's characteristic function which vanishes for $y=x$ is given by

$$
\begin{aligned}
W=2 \nu[(\sinh \lambda \cosh \lambda & +\lambda)-(\sinh \mu \cosh \mu+\mu)] \\
& =2 \nu[\sinh (\lambda-\mu) \cosh (\lambda+\mu)+(\lambda-\mu)] .
\end{aligned}
$$

Consistent with (35), we have $\lambda \geqslant \mu \geqslant 0$. As defined, $\lambda, \mu$, and $v$ are real for hyperbolic orbits (positive energy) and pure imaginary for elliptical orbits (negative energy). 
We turn next to the time-dependent Hamilton-Jacobi equation for Hamilton's principal function $S\left(\mathbf{r}_{1}, \mathbf{r}_{2}, t\right)$ :

$$
\partial S / \partial t+\frac{1}{2}\left(\nabla_{1} S\right)^{2}-Z / r_{1}=0
$$

with the analog for $\mathbf{r}_{2}$. By the same line of reasoning that led to Eqs. (36) we obtain

$$
\frac{1}{2} S_{t}+S_{x}^{2}-Z / x=0, \quad \frac{1}{2} S_{t}+S_{y}^{2}-Z / y=0 .
$$

The solution of (41) was given some time ago [22]. A simpler approach can be based on the Legendre transformation

$$
S(x, y, t) \equiv W(x, y, E)-E t .
$$

The time variable is found from

$$
t=\frac{\partial W}{\partial E}=-\frac{v^{3}}{Z^{2}} \frac{\partial W}{\partial v} .
$$

Taking account of the dependence of $\lambda$ and $\mu$ on $\nu$ through (37), we obtain

$$
\begin{aligned}
Z^{2} t=2 \nu^{3}[(\sinh \lambda \cosh \lambda-\lambda)-(\sinh \mu \cosh \mu-\mu)] \\
=2 \nu^{3}[\sinh (\lambda-\mu) \cosh (\lambda+\mu)-(\lambda-\mu)] .
\end{aligned}
$$

This is equivalent to a famous result in planetary astronomy, Lambert's theorem, published in 1761. According to the theorem, the transit time between two points in a planetary orbit is given by [23]

$$
\begin{aligned}
& t=\frac{\tau}{\pi}[(\alpha-\sin \alpha)-(\beta-\sin \beta)], \\
& \qquad \sin \frac{\alpha}{2}=\left(\frac{x}{4 a}\right)^{1 / 2}, \quad \sin \frac{\beta}{2}=\left(\frac{y}{4 a}\right)^{1 / 2}
\end{aligned}
$$

where $\tau$ is the orbital period and $a$ the semimajor axis.

The variables $\lambda, \mu, v$ can now be regarded as implicit functions of $x, y, t$ in the network of relationships expressed by Eqs. (37) and (44). These are further enumerated in Appendix A. Substituting into (42), we obtain finally the requisite solution for Hamilton's principal function:

$$
S=\nu[\sinh (\lambda-\mu) \cosh (\lambda+\mu)+3(\lambda-\mu)] .
$$

\section{Path-Integral Representation of the Coulomb Green's Function}

A key element in Hostler's [4] derivation of the Coulomb Green's function was an integral representation for a products of two Whittaker functions given by Buchholz [24]. With the appropriate specialization of variables, $G^{+}$can be reduced to the following integral form [25]:

$$
G^{+}(x, y, k)=\frac{i k}{2 \pi} \int_{0}^{\infty} d s \sinh s\left(\operatorname{coth} \frac{s}{2}\right)^{2 i \nu} e^{i k \xi \cosh s} J_{0}(k \eta \sinh s)
$$


with

$$
\xi \equiv \frac{1}{2}(x+y), \quad \eta \equiv(x y)^{1 / 2}, \quad \nu \equiv Z / k .
$$

The substitution $\sinh s=\operatorname{csch} q$ transforms the integral to

$$
G^{+}(x, y, k)=\frac{i k}{2 \pi} \int_{0}^{\infty} d q \operatorname{csch}^{2} q e^{2 i v q} e^{i k \xi \operatorname{coth} q} J_{0}(k \eta \operatorname{csch} q) .
$$

By Kummer's second formula [26], the Bessel function can be written

$$
J_{0}(z)=e^{-i z} M\left(\frac{1}{2}, 1,2 i z\right)
$$

where $M(a, b, z)$ is a confluent hypergeometric function [cf. Eq. (8)]. Thus the integrand in (49) contains a factor of the form $e^{i w}$ with

$$
W(q)=2 v q+k \xi \operatorname{coth} q-k \eta \operatorname{csch} q .
$$

Let us assume that this function is stationary for the value $q=q_{0}$, i.e.,

$$
W^{\prime}\left(q_{0}\right)=2 v-k \xi \operatorname{csch}^{2} q_{0}+k \eta \operatorname{csch} q_{0} \operatorname{coth} q_{0}=0 .
$$

Applying Eq. (Al) to the variables in (48) we have

$$
\begin{aligned}
k \xi / 2 \nu & =\sinh ^{2} \lambda+\sinh ^{2} \mu=\cosh (\lambda+\mu) \cosh (\lambda-\mu)-1, \\
k \eta / 2 \nu & =2 \sinh \lambda \sinh \mu=\cosh (\lambda+\mu)-\cosh (\lambda-\mu) .
\end{aligned}
$$

Equations (52) with (53) imply that

$$
q_{0}=\lambda-\mu,
$$

and thus

$$
W\left(q_{0}\right)=2 v[\sinh (\lambda-\mu) \cosh (\lambda+\mu)+(\lambda-\mu)]=W(x, y, E),
$$

which is Hamilton's characteristic function (39). Equation (49), in the form

$$
G^{+}(x, y, k)=\frac{i k}{2 \pi} \int_{0}^{\infty} d q \operatorname{csch}^{2} q M\left(\frac{1}{2}, 1,2 i k \eta \operatorname{csch} q\right) e^{i W(q)},
$$

can thereby be interpreted as a path-integral representation of the Green's function [27]. In this instance, the path is explicitly parametrized by a single variable $q$. The action function $W(q)$ assumes a stationary value for the classically allowed trajectory, with $q=q_{0}$. The path-integral formalism has recently been applied to the hydrogen atom by Duru and Kleinert [28] and other workers [29].

With the factor $e^{i W\left(q_{0}\right)}$ taken outside the integral (56), the Coulomb Green's function exhibits a structure characteristic of a semiclassical approximation [30]:

$$
G^{+}(x, y, E)=F(x, y, E) e^{i w(x, y, E)} .
$$

\section{Sturmian Propagators}

We consider again the integral formula (49) for the Coulomb Green's function. Analogous representations for the $\mathrm{N}$-dimensional free particle and harmonic oscillator have been shown to exhibit the structure of Fourier transforms. The 
corresponding time-dependent propagators are thus obtained almost by inspection [31]. In the case of (49), the Fourier transform involves the quantum number variable $v$ rather than the time and leads instead to a new type of propagator. More explicitly, we write

$$
G^{+}(x, y, v)=-\frac{2 i}{k} \int_{0}^{\infty} S_{c}(x, y, q) e^{2 i v q} d q
$$

with

$$
S_{c}(x, y, q)=-\left(k^{2} / 4 \pi\right) \operatorname{csch}^{2} q e^{i k \xi \operatorname{coth} q} J_{0}(k \eta \operatorname{csch} q) .
$$

The function (59), with $k$ and $q$ real, pertains to the Coulomb continuum. Of greater interest is the analog for the discrete spectrum, obtained by the substitutions $k \rightarrow i k, q \rightarrow i q$, viz.,

$$
S(x, y, q)=-\left(k^{2} / 4 \pi\right) \csc ^{2} q e^{i k \xi \cot q} J_{0}(k \eta \csc q) .
$$

We can write, in analogy with (2),

$$
S(x, y, q)=-\frac{1}{\pi(x-y)}\left(\frac{\partial}{\partial x}-\frac{\partial}{\partial y}\right) s(x, y, q) .
$$

Using the identity $(\partial / \partial z) z J_{1}(z)=z J_{0}(z)$, we find

$$
s(x, y, q)=-\frac{1}{2} k \eta \csc q e^{i k \xi \cot q} J_{1}(k \eta \csc q) .
$$

A spectral representation of the function (62) follows from the Hille-Hardy formula [33]:

$$
\begin{aligned}
& \frac{e^{(x+y) h /(1+h)}}{1+h} \frac{J_{\mu}\left[2(x y h)^{1 / 2} /(1+h)\right]}{(x y h)^{\mu / 2}} \\
& =\sum_{\lambda=0}^{\infty} \frac{\lambda !}{\Gamma(\lambda+\mu+1)}(-h)^{\lambda} L_{\lambda}^{(\mu)}(x) L_{\lambda}^{(\mu)}(y) .
\end{aligned}
$$

In (63), let $\mu=1, x \rightarrow k x, y \rightarrow k y, \lambda=n-1, h=-e^{-2 i q}$, and express the Laguerre functions $L_{\lambda}^{(\mu)}$ in terms of Whittaker functions. The result is

$$
s(x, y, q)=\sum_{n=1}^{\infty} n M_{n}^{1 / 2}(k x) M_{n}^{1 / 2}(k y) e^{-2 i n q} .
$$

Making use of $(20)-(23)$, we find that $S(x, y, q)$ is represented by the summation

$$
S\left(\mathbf{r}_{1}, \mathbf{r}_{2}, q\right)=\sum_{n ! m} \phi_{n l m}\left(\mathbf{r}_{1}\right) \phi_{n l m}^{*}\left(\mathbf{r}_{2}\right) e^{-2 i n q}
$$

in terms of the hydrogenlike functions

$$
\phi_{n l m}(\mathbf{r})=[(n+l) ! /(n-l-1) !]^{1 / 2} r^{-1} M_{n}^{l+1 / 2}(2 k r) Y_{l m}(\theta, \phi) .
$$

The latter differ from the hydrogenic eigenfunctions (20) in that the scaling constants $k$ have a fixed value for all $n$, and are not individually equal to $Z / n$. The $\phi(\mathbf{r})$ have been dubbed "Sturmian functions" [34]. These have found ex- 
tensive applications in atomic and molecular computations.

The structure of $S\left(\mathbf{r}_{1}, \mathbf{r}_{2}, q\right)$ suggests its designation as a "Sturmian propagator" [32]. Whereas the discrete hydrogenic eigenfunctions must be augmented by a continuum to form a complete set, a denumerable set of Sturmian functions is complete over the same domain. This can be shown directly from the $q \rightarrow 0$ limit of the Sturmian propagator (60). Using the asymptotic form of the Bessel function for large values of its argument, we find

$$
S\left(\mathbf{r}_{1}, \mathbf{r}_{2}, 0\right)=\sum_{n l m} \phi_{n l m}\left(\mathbf{r}_{1}\right) \phi^{*}{ }_{n l m}\left(\mathbf{r}_{2}\right)=\left(r_{1} r_{2}\right)^{1 / 2} \delta\left(\mathbf{r}_{1}-\mathbf{r}_{2}\right) .
$$

This represents the closure condition on the set $\left\{\phi_{n l m}\right\}$. The factor $\left(r_{1} r_{2}\right)^{1 / 2}$ arises from the weight factor $r$ (rather than $r^{2}$ ) in the orthonormalization of the radial functions in (66).

The positive-energy Sturmian propagator (59) can analogously be shown to represent the summation

$$
S_{c}\left(\mathbf{r}_{1}, \mathbf{r}_{2}, q\right)=\sum_{l m} \int_{0}^{\infty} d \nu \phi_{v l m}\left(\mathbf{r}_{1}\right) \phi_{\nu l m}^{*}\left(\mathbf{r}_{2}\right) e^{-2 i v q}
$$

in terms of a complete set of continuum Sturmian functions

$$
\phi_{\nu l m}(\mathbf{r})=(2 \pi)^{-1 / 2} i^{l+1} e^{\pi \nu / 2} \Gamma(l+1-i v) r^{-1} M_{i \nu}^{l+1 / 2}(-2 i k r) Y_{l m}(\theta, \phi) .
$$

Just as in the case of time-dependent Green's functions, the Sturmian propagator represents a solution of a partial differential equation [32]. By Fourier transformation of Eq. (1) using (58) it follows that

$$
\frac{k^{2}}{2} S+\frac{1}{2} \nabla_{1}^{2} S+\frac{i k}{2 r_{1}} \frac{\partial S}{\partial q}=0
$$

subject to the boundary condition $S\left(\mathbf{r}_{1}, \mathbf{r}_{2}, 0\right)=\left(r_{1} r_{2}\right)^{1 / 2} \delta\left(\mathbf{r}_{1}-\mathbf{r}_{2}\right)$.

It is shown in [31] that, for an $N$-dimensional Coulomb system, the radial Sturmian propagator for the $L$ th partial wave is given by

$$
\begin{aligned}
S_{L}^{(N)}\left(r_{1}, r_{2}, q\right)=(-i)^{2 L+N-1} k\left(r_{1} r_{2}\right)^{1-N / 2} \csc q \\
\quad \times e^{i k\left(r_{1}+r_{2}\right) \cot J_{2 \mathcal{L}+N-2}\left[2 k\left(r_{1} r_{2}\right)^{1 / 2} \csc q\right] .}
\end{aligned}
$$

This is related to the $\lambda$ th partial-wave radial propagator for a $\nu$-dimensional harmonic oscillator by

$$
\left(r_{1} r_{2}\right)^{N / 2-1} S_{L}^{(N)}\left(r_{1}, r_{2}, q\right)=\left(\rho_{1} \rho_{2}\right)^{v / 2-1} K_{\lambda}^{(v)}\left(\rho_{1}, \rho_{2}, t\right)
$$

such that $2 L+N-2=\lambda+\frac{1}{2} \nu-1$. The well-known connection between Coulomb and oscillator eigenstates of various dimensionality [35] is thus manifested in a new way.

\section{The Coulomb Propagator}

Feynman's path-integral formulation of quantum mechanics [27], despite its notable successes [36], remains incomplete with regard to two elementary aspects: the representation of spinors and the hydrogen atom. Although the path 
integration for the Coulomb problem has been carried out with the aid of the Kustaanheimo-Stiefel transformation [37], no explicit form for the propagator $K\left(\mathbf{r}_{1}, \mathbf{r}_{2}, t\right)$ has resulted as yet. We note that a number of integral representations [38], numerical solutions [39], and asymptotic forms [40] related to $K$ have been found.

The propagator in Feynman's formalism is represented by the path integral

$$
K\left(\mathbf{r}_{1}, \mathbf{r}_{2}, t\right)=\int \mathscr{P}[\mathbf{r}] \exp \left(\frac{i}{\hbar} \int_{0}^{t} L(\mathbf{r}, \dot{\mathbf{r}}) d t\right),
$$

in which $L$ is the classical Lagrangian. Equivalently, the Coulomb propagator is the solution of the time-dependent Schrödinger equation

$$
i \frac{\partial K}{\partial t}+\frac{1}{2} \nabla_{1}^{2} K+\frac{Z}{r_{1}} K=0
$$

subject to the initial condition

$$
K\left(\mathbf{r}_{1}, \mathbf{r}_{2}, 0\right)=\delta\left(\mathbf{r}_{1}-\mathbf{r}_{2}\right) .
$$

The propagator is related to the time-independent Green's function by a Fourier transform [41]

$$
G^{+}=-i \int_{0}^{\infty} K e^{i E t} d t
$$

Since, as discussed above, $G$ depends on $\mathbf{r}_{1}$ and $\mathbf{r}_{2}$ through the combinations $x$ and $y$ [cf. Eq. (4)], $K$ must also. Taking $K=K(x, y, t)$, the sum and difference of Eq. (74) with its analog in $\mathbf{r}_{2}$ leads, after some algebra, to

$$
\begin{aligned}
i K_{t}+K_{x x}+K_{y y}+[2 /(x-y)]\left(K_{x}-K_{y}\right) \\
+[(x+y) / x y]\left(K_{x}+K_{y}+Z K\right)=0
\end{aligned}
$$

( $\Sigma$-equation)

and

$$
\begin{aligned}
x y\left(K_{y y}-K_{x x}\right)+ & (x-y)\left(K_{x}+K_{y}+Z K\right)=0 \\
& (\Delta \text {-equation })
\end{aligned}
$$

If we assume a structure analogous to that of the Green's function in Eq. (2),

$$
K(x, y, t)=-\frac{1}{\pi(x-y)}\left(\frac{\partial}{\partial x}-\frac{\partial}{\partial y}\right) k(x, y, t),
$$

then Eqs. (77)-(78) are equivalent to

$$
\frac{1}{2} i k_{t}+k_{x x}+(Z / x) k=0, \quad \frac{1}{2} i k_{t}+k_{y y}+(Z / y) k=0 .
$$

This is analogous to the reduction of the Hamilton-Jacobi equation to Eqs. (41).

In the limit $Z \rightarrow 0, K$ becomes the free-particle propagator

$$
\begin{aligned}
K^{0}(x, y, t) & =(2 \pi i t)^{-3 / 2} e^{i(x-y) / 8 t} \\
& =(2 \pi i t)^{-3 / 2} e^{i r_{12}^{2} / 2 t},
\end{aligned}
$$


and correspondingly

$$
k^{0}(x, y, t)=(2 \pi i t)^{-1 / 2} e^{i(x-y)^{2} / 8 t} .
$$

As shown by Feynman [27] and others [42], for Lagrangians expressible as quadratic forms in generalized coordinates and velocities, the propagator assumes the simple structure

$$
K\left(\mathbf{r}_{1}, \mathbf{r}_{2}, t\right)=F(t) e^{i S\left(\mathbf{r}_{1}, \mathbf{r}_{2}, t\right) / \hbar},
$$

in which $S$ is Hamilton's principal function (now loosely called the "action"). Given $S$, the preexponential factor $F(t)$ is easily determined from Eqs. (74)(75). For nonharmonic potentials, including the Coulomb problem, the decomposition (83) is no longer exact.

Let us write $k(x, y, t)$ in Eq. (80) in the form

$$
k(x, y, t)=f(x, y, t) e^{i S(x, y, t) / \hbar}
$$

with the preexponential factor now a function of $x$ and $y$, as well as $t$. After reintroducing $\hbar$ into (80), for use as an expansion parameter, we substitute (84) and collect powers of $\hbar$. The result is

$$
-\left(\frac{1}{2} S_{t}+S_{x}^{2}-Z / x\right) f+i \hbar\left(\frac{1}{2} f_{t}+2 S_{x} f_{x}+S_{x x} f\right)+\hbar^{2} f_{x x}=0 .
$$

Within the semiclassical approximation [43], the term in $\hbar^{2}$ is neglected. The terms independent of $\hbar$ represent the Hamilton-Jacobi equation, which has been solved above. The segment of first order in $\hbar$ gives

$$
\frac{1}{2} f_{t}+2 S_{x} f_{x}+S_{x x} f=0,
$$

with an analogous equation in $y$. Using formulas derived in the Appendix, we can reexpress Eq. (86) in terms of the auxilliary variables $\lambda, \mu, \nu$. From Eqs. (A5) and (A6) there follows a near-miraculous reduction of (86) to an ordinary differential equation:

$$
\frac{\partial f}{\partial \lambda}+\frac{1}{2}\left(\frac{J_{\lambda}}{J}-\frac{\cosh \lambda}{\sinh \lambda}\right) f=0 .
$$

The solution is

$$
f(\lambda, \mu, \nu)=[\sinh \lambda / J(\lambda, \mu)]^{1 / 2}(\text { function of } \mu, v) .
$$

From the analogous equation in $y$ and the reduction of $k$ to $k^{0}$ as $Z \rightarrow 0$ [cf. Eq. (82)], we find

$$
f(\lambda, \mu, \nu)=\left(Z^{2} / 4 \pi i\right)^{1 / 2} \nu^{-3 / 2}[\sinh \lambda \sinh \mu / J(\lambda, \mu)]^{1 / 2} .
$$

We obtain thus, in the semiclassical approximation,

$$
k\left(\mathbf{r}_{1}, \mathbf{r}_{2}, t\right) \approx f(\lambda, \mu, \nu) e^{i s(\lambda, \mu, \nu)}
$$

with $S$ given by (46). Finally, the propagator $K$ is found using Eq. (79). This can also be expressed in the form [44]

$$
K\left(\mathbf{r}_{1}, \mathbf{r}_{2}, t\right) \approx F(\lambda, \mu, \nu) e^{i S(\lambda, \mu, \nu)}
$$


with

$$
\begin{aligned}
F(\lambda, \mu, v)=\frac{1}{2}\left(Z^{2} / 4 \pi i\right)^{3 / 2} \nu^{-9 / 2}[\sinh (\lambda-\mu)]^{-1} & \\
& \times[\sinh \lambda \sinh (\mu) J(\lambda, \mu)]^{-1 / 2} .
\end{aligned}
$$

The Coulomb propagator reduces to the free-particle form (81) as $\lambda, \mu \rightarrow \infty$, corresponding to any of the limits $Z \rightarrow 0 ; x, y \rightarrow \infty$, or $t \rightarrow 0$.

The semiclassical approximation (91)-(92) has two important properties in common with the exact Coulomb propagator. First, it correctly reduces to a delta function for $t=0$, consistent with the initial condition (75). Also, it satisfies the closure condition on propagators, viz.,

$$
\int K\left(\mathbf{r}_{1}, \mathbf{r}_{2}, t_{2}-t_{1}\right) K\left(\mathbf{r}_{2}, \mathbf{r}_{3}, t_{3}-t_{2}\right) d^{3} \mathbf{r}_{2}=K\left(\mathbf{r}_{1}, \mathbf{r}_{3}, t_{3}-t_{1}\right)
$$

By virtue of these properties, the semiclassical Coulomb propagator becomes applicable in a formalism we proposed some time ago $[45,46]$ for aufbau of approximate many-electron Green's functions and analysis of their eigenvalue spectra.

\section{Appendix: Transformation Formulas for $\lambda, \mu, \nu$ Variables}

The auxilliary variables introduced in the solution of the Hamilton-Jacobi equation are related to $x, y, t$ through

$$
\begin{aligned}
Z x=4 v^{2} \sinh ^{2} \lambda, \quad Z y & =4 v^{2} \sinh ^{2} \mu, \\
Z^{2} t & =2 v^{3}[\sinh (\lambda-\mu) \cosh (\lambda+\mu)-(\lambda-\mu)] .
\end{aligned}
$$

To express derivatives with respect to $x, y, t$ in terms of $\lambda, \mu, v$, the elements of the Jacobian matrix $\partial(\lambda, \mu, \nu) / \partial(x, y, t)$ are required. These are enumerated in the following array:

\begin{tabular}{clll} 
& \multicolumn{1}{c}{$x$} & \multicolumn{1}{c}{$y$} & \multicolumn{1}{c}{$t$} \\
\hline$\lambda$ & $\left(3 C_{\mu} T+2 S_{\mu}^{3}\right) / 8 \nu^{2} S_{\lambda} J$ & $-S_{\lambda} S_{\mu} / 4 \nu^{2} J$ & $-S_{\lambda} C_{\mu} / 2 \nu^{3} J$ \\
$\mu$ & $S_{\lambda} S_{\mu} / 4 v^{2} J$ & $\left(3 C_{\lambda} T-2 S_{\lambda}^{3} / 8 \nu^{2} S_{\mu} J\right.$ & $-C_{\lambda} S_{\mu} / 2 \nu^{3} J$ \\
$\nu$ & $-S_{\lambda} C_{\mu} / 4 \nu J$ & $C_{\lambda} S_{\mu} / 4 \nu J$ & $C_{\lambda} C_{\mu} / 2 \nu^{2} J$
\end{tabular}

The following abbreviations have been employed: $S_{\lambda}=\sinh \lambda, C_{\lambda}=\cosh \lambda$, $S_{\mu}=\sinh \mu, C_{\mu}=\cosh \mu$. Also we have defined

$$
\begin{aligned}
j(\lambda) & \equiv S_{\lambda}^{3}+3 S_{\lambda}-3 \lambda C_{\lambda}, \\
J(\lambda, \mu) & \equiv C_{\mu} j(\lambda)-C_{\lambda} j(\mu), \\
T(\lambda, \mu) & \equiv\left(S_{\lambda} C_{\lambda}-\lambda\right)-\left(S_{\mu} C_{\mu}-\mu\right) .
\end{aligned}
$$

Now the following identities are readily verified:

$$
C_{\lambda} j^{\prime}(\lambda)=S_{\lambda} j(\lambda)+2 S_{\lambda}^{4}, \quad C_{\lambda} \frac{\partial J}{\partial \lambda}=S_{\lambda} J(\lambda, \mu)+2 S_{\lambda}^{4} C_{\mu} .
$$

The required derivatives of $S$ are evaluated by application of the above formulas:

$$
S_{x}=(2 v)^{-1} \operatorname{coth} \lambda, \quad S_{y}=-(2 v)^{-1} \operatorname{coth} \mu .
$$


Also

$$
S_{x x}=\frac{1}{16 v^{3} S_{\lambda}^{3} C_{\mu}}\left(\frac{2 S_{\lambda}^{5} C_{\mu}}{J}-1\right)=\frac{1}{16 v^{3} S_{\lambda}^{2}}\left(\frac{J_{\lambda}}{J}-\frac{C_{\lambda}}{S_{\lambda}}\right) .
$$

We conclude with the remarkable operator relation

$$
\frac{1}{2} \frac{\partial}{\partial t}+2 S_{x} \frac{\partial}{\partial x}=\frac{1}{8 \nu^{3} S_{\lambda}^{2}} \frac{\partial}{\partial \lambda} .
$$

\section{Bibliography}

[1] J. Meixner, Math. Z. 36, 677 (1933).

[2] R. A. Mapleton, Phys. Rev, 117, 479 (1960); J. Math. Phys. 2, 478 (1961); K. Mano, J. Math. Phys. 4, 22 (1963); 5, 505 (1964); E. H. Wichmann and C. H. Woo, J. Math. Phys. 2, 178 (1961); H. F. Hameka, J. Chem. Phys. 47, 2728 (1967).

[3] J. Schwinger, J. Math. Phys. 5, 1606 (1964).

[4] L. Hostler and R. H. Pratt, Phys. Rev. Lett. 10, 469 (1963); L. Hostler, J. Math. Phys. 5, 591 (1964).

[5] S. M. Blinder, J. Math. Phys. 22, 306 (1981).

[6] H. Buchholz, The Confluent Hypergeometric Function (Springer, New York, 1969).

[7] See, for example, R. Gilmore, Lie Groups, Lie Algebras, and Some of Their Applications (Wiley, New York, 1974), pp. 428ff.

[8] L. Hostler, J. Math. Phys. 8, 642 (1967).

[9] X. Shi and S. M. Blinder, Chem. Phys. Lett. 104, 572 (1984).

[10] E. Hylleraas, Adv. Quantum Chem. 1, 1 (1964).

[11] E. Fermi, Z. Phys. 60, 320 (1930); S. M. Blinder, Adv. Quantum Chem. 2, 47 (1965).

[12] C. Schwartz, Ann. Phys. (N.Y.) 6, 156 (1959).

[13] S. M. Blinder, Phys. Rev. A 18, 853 (1978); Theor. Chim. Acta 53, 159 (1979).

[14] T. Hannesson and S. M. Blinder, Int. J. Quantum Chem. 15, 7 (1979).

[15] T. Hannesson and S. M. Blinder, Bull. Am. Phys. Soc. 25, 171 (1980).

[16] T. P. Das and R. Bersohn, Phys. Rev. 115, 897 (1959); H. J. Kolker and M. Karplus, J. Chem. Phys. 41, 1259 (1964); J. Schäfer and R. Yaris, J. Chem. Phys. 46, 948 (1967); H. Wrubel and J. Voitländer, Mol. Phys. 25, 323 (1973).

[17] E. Ishiguro, Phys. Rev. 111, 203 (1958).

[18] S. M. Blinder, Bull. Am. Phys. Soc. 28, 1309 (1983).

[19] A. Unsöld, Ann. Phys. 82, 355 (1927).

[20] See, for example, J. L. Synge, "Classical Dynamics," in Handbuch der Physik, Vol. III/1, S. Flügge, Ed. (Springer, Berlin, 1960), pp. 117ff.

[21] C. Runge, Vector Analysis (Dutton, New York, 1919), p. 79; W. Lenz, Z. Phys. 24, 197 (1924); W. Pauli, Z. Phys. 36, 336 (1926) [English translation in B. L. van der Waerden, Sources of Quantum Mechanics (Dover, New York, 1968), p. 387].

[22] S. M. Blinder, J. Math. Phys. 16, 2000 (1975).

[23] E. T. Whittaker, A Treatise on the Analytical Dynamics of Particles and Rigid Bodies, 4th ed. (Cambridge University, Cambridge, 1965) pp. 91-92.

[24] H. Buchholz, The Confluent Hypergeometric Function (Springer, New York, 1969), p. 86; Eq. $(5 c)$.

[25] S. M. Blinder, J. Math. Phys. 22, 310 (1981), Eq. (3.25)

[26] M. Abramowitz and I. A. Stegun, Eds., Handbook of Mathematical Functions (National Bureau of Standards, Washington, D.C., 1972), p. 362, Eq. (9.1.69).

[27] R. P. Feynman, Rev. Mod. Phys. 20, 367 (1948); R. P. Feynman and A. R. Hibbs, Quantum Mechanics and Path Integrals (McGraw-Hill, New York, 1965).

[28] I. H. Duru and H. Kleinert, Phys. Lett. 84B 185 (1979); Fortschr. Phys. 30, 401 (1982). 
[29] M. C. Gutzwiller, J. Math. Phys. 8, 1979 (1967); C. Gerry and A. Inomata, Phys. Lett. 84A, 172 (1981); R. Ho and A. Inomata, Phys. Rev. Lett. 48, 231 (1982); H. Grinberg, J. Marafion, and H. Vucetich, J. Chem. Phys. 78, 839 (1983); Int. J. Quantum Chem. 23, 379 (1983).

[30] S. M. Blinder, Bull. Am. Phys. Soc. 26, 41 (1981).

[31] S. M. Blinder, J. Math. Phys. 25, 905 (1984).

[32] S. M. Blinder, Phys. Rev. A 29, 1674 (1984).

[33] H. Buchholz, The Confluent Hypergeometric Function (Springer, New York, 1969), p. 139, Eq. (12a); A. Erdelyi, Ed., Higher Transcendental Functions (McGraw-Hill, New York, 1953), Vol. 2, p. 189.

[34] M. Rotenberg, Ann. Phys. (N.Y.) 19, 262 (1962); Adv. At. Mol. Phys. 6, 233 (1970).

[35] A. Giovannini and T. Tonietteti, Nuovo Cimento A54, 1 (1968).

[36] R. P. Feynman, Phys. Rev. 80, 440 (1950); G. J. Papadopoulos and J. T. Devreese, Eds., Path Integrals and Their Application in Quantum Statistical and Solid State Physics (Plenum, New York, 1978); W. H. Miller and T. F. George, J. Chem. Phys. 56, 5637 (1972); W. H. Miller, Adv. Chem. Phys. 25, 69 (1974).

[37] P. Kustaanheimo and E. Stiefel, J. Reine Angew. Math. 218, 204 (1965).

[38] M. J. Goovaerts and J. T. Devreese, J. Math. Phys. 13, 1070 (1972); M. J. Goovaerts, F. Broeckx, and P. Van Camp, Physica 64, 47 (1973).

[39] R. G. Storer, J. Math. Phys. 9, 964 (1968).

[40] S. M. Blinder, Int. J. Quantum Chem. S14, 43 (1980).

[41] See, for example, S. M. Blinder, Foundations of Quantum Dynamics (Academic, London, 1974), Chap. 6.

[42] S. Albeverio and R. Høegh-Krohn, "Mathematical Theory of Feynman Path Integrals," in Lecture Notes in Mathematics 523 (Springer, New York, 1976).

[43] J. Linderberg and Y. Ohrn, Propagators in Quantum Chemistry (Academic, London, 1973), pp. 34ff; N. Froman, in Semiclassical Methods in Molecular Scattering and Spectroscopy, M. S. Child, Ed. (Reidel, Dordrecht, 1980), pp. 1-44.

[44] S. M. Blinder, Phys. Rev. Lett. 52, 1771 (1984).

[45] S. M. Blinder, International Review of Science, Vol. I, Theoretical Chemistry (Butterworths, London, 1975).

[46] S. M. Blinder, Theor. Chim. Acta 24, 382 (1972).

Received June 18, 1984 